\title{
Study on diurnal variation in TSH and freeT4 levels of healthy adults
}

\author{
Liyanage $\mathrm{YSH}^{1}$, Siriwardhana $\mathrm{ID}^{2}$, Dissanayake $\mathrm{M}^{3}$, Dayanath BKPT ${ }^{4}$
}

${ }^{1}$ Allied Health Sciences Degree Program, Faculty of Medicine, University of Ruhuna, Galle, Sri Lanka

2 Department of Pathology, Faculty of Medicine, University of Ruhuna, Galle, Sri Lanka

${ }^{3}$ Department of Pathology, Teaching Hospital Karapitiya Galle, Sri Lanka

${ }^{4}$ Department of Pathology, North Colombo Teaching Hospital, Ragama Sri Lanka

\section{Abstract}

Thyroid stimulating hormone (TSH) secretion shows a circadian rhythm, affecting free thyroxine (fT4) secretion in the same manner. Samples are collected randomly throughout the day for serum TSH \& fT4 measurement in current practice. This may affect interpretation of results if the follow up samples are collected at different times of the day from the same individual. We aimed to assess the diurnal variation in serum TSH and fT4 levels in healthy adults. Healthy adult volunteers with Sinhalese ethnicity aged $21-50$ years were selected for the study. Subjects with thyroid or any other disease, on medications including oral contraceptives and postmenopausal women were excluded. Two blood samples were drawn from each subject at 8-9 am and 3-4 pm on the same day. TSH and fT4 were measured using VITROS ECi Immunodiagnostic system. Paired $\mathrm{T}$ test was used to assess whether there was a statistically significant diurnal variation in hormone values. Data were analyzed using IBM SPSS Statistics Version 20 and Microsoft Excel 2013. A total number of 36 subjects with an equal number of males \& females participated in the study. There was no variation in hormone levels due to age or gender. Log transformed TSH and fT4, showed a statistically significant difference for both TSH $(\mathrm{p}=0.013)$ and freeT4 $(\mathrm{p}<0.001)$ values in the morning and afternoon. Standardization of sample collection time may be important for TSH and fT4 hormone assays as this study revealed a statistically significant diurnal variation for both hormones. However further studies are needed in patients with thyroid diseases and individuals of other age groups as well to ascertain the clinical significance.

DOI: http://doi.org/10.4038/sjem.v8i1.7346

Received: 18 $8^{\text {th }}$ of February $2018 \quad$ Accepted revised version: $7^{\text {th }}$ of March $2018 \quad$ Published: $9^{\text {th }}$ April 2018

Correspondence: e-mail: dissanyake@gmail.com

This is an open-access article distributed under the terms of the Creative Commons Attribution 4.0 International License, which permits unrestricted use, distribution and reproduction in any medium provided the original author and source are credited 


\section{Introduction}

Thyroxine $\left(\mathrm{T}_{4}\right)$ and triiodothyronine $\left(\mathrm{T}_{3}\right)$ hormones are produced by the thyroid gland. $\mathrm{T}_{4}$ is the pro-hormone of $T_{3}$ which is the active form. $T_{4}$ production is much higher than the $\mathrm{T}_{3}$ production. In the thyroid gland about $40 \%$ of the $\mathrm{T}_{4}$ converts to $\mathrm{T}_{3}$ by deiodinase enzyme at cellular level. In plasma more than $99 \%$ of $\mathrm{T}_{3}$ and $\mathrm{T}_{4}$ are bound to hormone binding proteins (albumin, thyroid binding pre-albumin and thyroid binding globulin). Hence, total hormone ( $\mathrm{T}_{4}$ and $\mathrm{T}_{3}$ ) levels change with the changes of binding protein levels due to various physiological and pathological conditions. The free fraction is the physiologically active form and independent of effects due to variation of binding proteins. Clinicians now prefer free $\mathrm{T}_{4}\left(\mathrm{fT}_{4}\right)$ and free $\mathrm{T}_{3}\left(\mathrm{fT}_{3}\right)$ levels over total hormone levels (1-4).

Thyrotropin Releasing Hormone (TRH) is synthesised in the hypothalamus and stimulates the secretion of thyroid stimulating hormone (TSH) from the anterior pituitary gland. TSH stimulates the growth and the activity of thyroid follicular cells which increase the release of $T_{3}$ and $T_{4}$. TRH and TSH secretion is controlled by negative feedback mechanism of $\mathrm{T}_{3}$ and $\mathrm{T}_{4}$. Some other hormones and drugs such as glucocorticoid, dopamine and somatostatin inhibit TSH secretion (1-4). The hypothalamus-pituitary- thyroid axis is the main regulator of TSH and $\mathrm{T}_{4}$ levels in body (4).

TSH and $\mathrm{fT}_{4}$ are the commonly used tests in evaluating the functional status of the thyroid gland. Patients come to the laboratory to test their serum TSH and $\mathrm{fT}_{4}$ levels throughout the day and specimens are collected without considering the time of the day. Hence, sample collection time is important if there is a significant diurnal variation in $\mathrm{TSH}$ and $\mathrm{fT}_{4}$ levels. in TSH and $\mathrm{fT}_{4}$ levels. For the TSH immunoassay test, serum or plasma can be used. If plasma is used, heparin or Ethylenediaminetetraacetic acid (EDTA) can be used as the anticoagulant. The sample should be free of haemolysis and lipaemia. Turbid samples cannot be used. Serum/plasma separated from the clot/red cells, is stable for 5-7 days at $2-8{ }^{\circ} \mathrm{C}$ and one month if frozen at $-20{ }^{\circ} \mathrm{C}$. Repeated freezing and thawing should be avoided (5).

Serum is the preferred sample for $\mathrm{fT}_{4}$, similarly for TSH. But plasma with EDTA or heparin can also be used. $\mathrm{T}_{4}$ is stable up to 7 days at room temperature, but the best is $2-8{ }^{\circ} \mathrm{C}$. Frozen $\left(\right.$ at $\left.-20{ }^{\circ} \mathrm{C}\right)$ specimens are stable up to 30 days. Repeated freezing and thawing should be avoided. Minor haemolysis and lipaemia have no significant effect on most $\mathrm{fT}_{4}$ immunoassays. However heavy haemolysis should be avoided as it can dilute the sample. Turbid samples should be centrifuged before testing, because turbidity may affect the test result. $\mathrm{T}_{4}$ auto-antibodies can interfere with the assay depending on the method $(4,6)$.

TSH secretion occurs in a circadian rhythm. Highest concentration occurs between 2.00 am to $4.00 \mathrm{am}$, whereas the lowest is seen in $5.00 \mathrm{pm}$ to $6.00 \mathrm{pm}$. Low amplitude oscillations occur throughout the day (4). This oscillation may be lost in critical illnesses and after surgery (4). There are no significant differences when race and sex are considered. TSH increases immediately after the birth and decline back to the cord blood level by three days (4). It reaches the adult range in the first week. According to some other authors, TSH secretion is pulsatile and the mean-output starts to rise at about $9.00 \mathrm{pm}$, peaks at midnight, and then declines during the day (3). The studies done to assess the diurnal variation in thyroid function tests aren't many and the conclusions drawn have no consensus. (Table 1)

Table 1: Review of previous studies

Study

Weeke J, Gundersen H J [7]
(1978

Sviridonova MA et al. [8] (2013)

\section{Conclusions}

- $\quad$ fT3 and TSH levels low during the day time \& high at night.

- The same tendency observed for fT4; not clinically significant.

- $\quad$ Sample collection done between 8.00 am to 9.00 am and $2.00 \mathrm{pm}$ to $4.00 \mathrm{pm}$.

- Median of morning TSH level in patients with subclinical hypothyroidism, 5.83 $\mathrm{mU} / \mathrm{L}$ whereas $3.79 \mathrm{mU} / \mathrm{L}$ in the afternoon.

- The range of above TSH circadian variability is $73 \%$.

- Median of morning TSH level in patients, who take L-thyroxine, was $3.27 \mathrm{mU} / \mathrm{L}$ whereas $2.18 \mathrm{mU} / \mathrm{L}$ in the afternoon. 
Lucke C et al. [9] (1977)

Weeke J Laurberg P [10] (1976)
- The range of above TSH circadian variability is $64.7 \%$.

- $\quad$ TSH peaks from $8.00 \mathrm{pm}$ to $2.00 \mathrm{am}$ and nadir from 7.00 am to $2.00 \mathrm{pm}$.

- Multiple, short-lived fluctuations observed for TSH.

- T4 levels were highest from 8.00 am to 12.00 noon and lowest from $11.00 \mathrm{pm}$ to $3.00 \mathrm{am}$.

- Multiple short-lived fluctuations observed for T4.

- TSH level low during day and high during the night, in mild hypothyroidism.

- No significant variation seen in serum T3 level.

- Patients with severe hypothyroidism showed no significant variation in both TSH and T3.

- Patients with severe hypothyroidism, treated, showed circadian periodicity in serum TSH levels.
The main objective of this study was to assess the diurnal variation in serum $\mathrm{TSH}$ and $\mathrm{fT}_{4}$ levels in healthy adults.

\section{Materials and Methodology}

This was an evaluation study and all specimens were analysed at the Department of Chemical Pathology, Teaching Hospital, Karapitiya. Ethical approval for the study was obtained from the Ethical Review Committee of Faculty of Medicine, University of Ruhuna, Sri Lanka. Convenient sampling was done on 36 individuals. Twelve individuals from each age group were selected (age 21 to 30, age 31 to 40, age 41 to 50). Each age group consisted of six males and six females. Healthy volunteers of Sinhalese ethnicity were invited to participate in the study. The study population comprised of the students and staff members of the Faculty of Medicine, University of Ruhuna and staff members of Teaching Hospital, Karapitiya. People with pregnancy, history of any illness, medications including oral contraceptive pills, menopause and age $<21$ and $>50$ years excluded from the study population. This study was done from June 2015 to February 2016. All the samples for serum $\mathrm{TSH}$ and $\mathrm{fT}_{4}$ were analysed on VITROS ECi Immunodiagnostic Systems, (Ortho Clinical Diagnostics USA).

Study participants were informed about the research project and written consent was obtained. Samples were collected from selected volunteers by a trained nursing students. Sample volume was equal to the volume of sample that collected for thyroid profile in routine setting $(2 \mathrm{ml})$. Venous blood samples were collected from the right median cubital vein. All individuals were given about 15 minutes to rest prior to sample collection. Samples were collected in the seated position. Tourniquet was used in all the sample collection procedures and tourniquet was not applied for more than one minute. Three cc syringes with 22-gauge needle were used to collect $2 \mathrm{ml}$ of sample into plain glass tubes. Collected samples were kept at room temperature for 30 minutes and serum was separated by centrifugation. Separated serum was aliquoted into new sample collection glass tubes. Serum was stored in the freezer at $-20{ }^{\circ} \mathrm{C}$ until they were analysed. Each individual underwent two sample collections on the same day between 8.00 am to $9.00 \mathrm{am}$ and $3.00 \mathrm{pm}$ to $4.00 \mathrm{pm}$. Standard quality management procedures including three levels of internal quality controls daily and monthly external quality assurance were implemented during the study period.

Morning and afternoon data were analysed separately and those data were compared with the reference ranges. The values taken from the 36 samples were analysed to see whether there were any outliers and to see whether the data distributed normally. Normality test results revealed that the data were not normally distributed. Using Resistant Rules for Outlier Labelling (11), two outliers were removed, and remaining 34 values were analysed to see whether the data were normally distributed. Even 
after removing outliers, the normality test results revealed that the data were skewed. So that $\log _{10}$ transformation was done for the remaining data to get rid of the skewness and make the data set normally distributed. This log values were used to apply paired $T$ - test, to identify whether there was any statistically significant variation between morning and afternoon data. A $p$-value of $<0.05$ was considered as statistically significant. For the data analysis IBM SPSS Statistics Version 20 and Microsoft Excel 2013 software packages were used.

Figure 1 - Diurnal Variation of TSH \& FT4 Levels

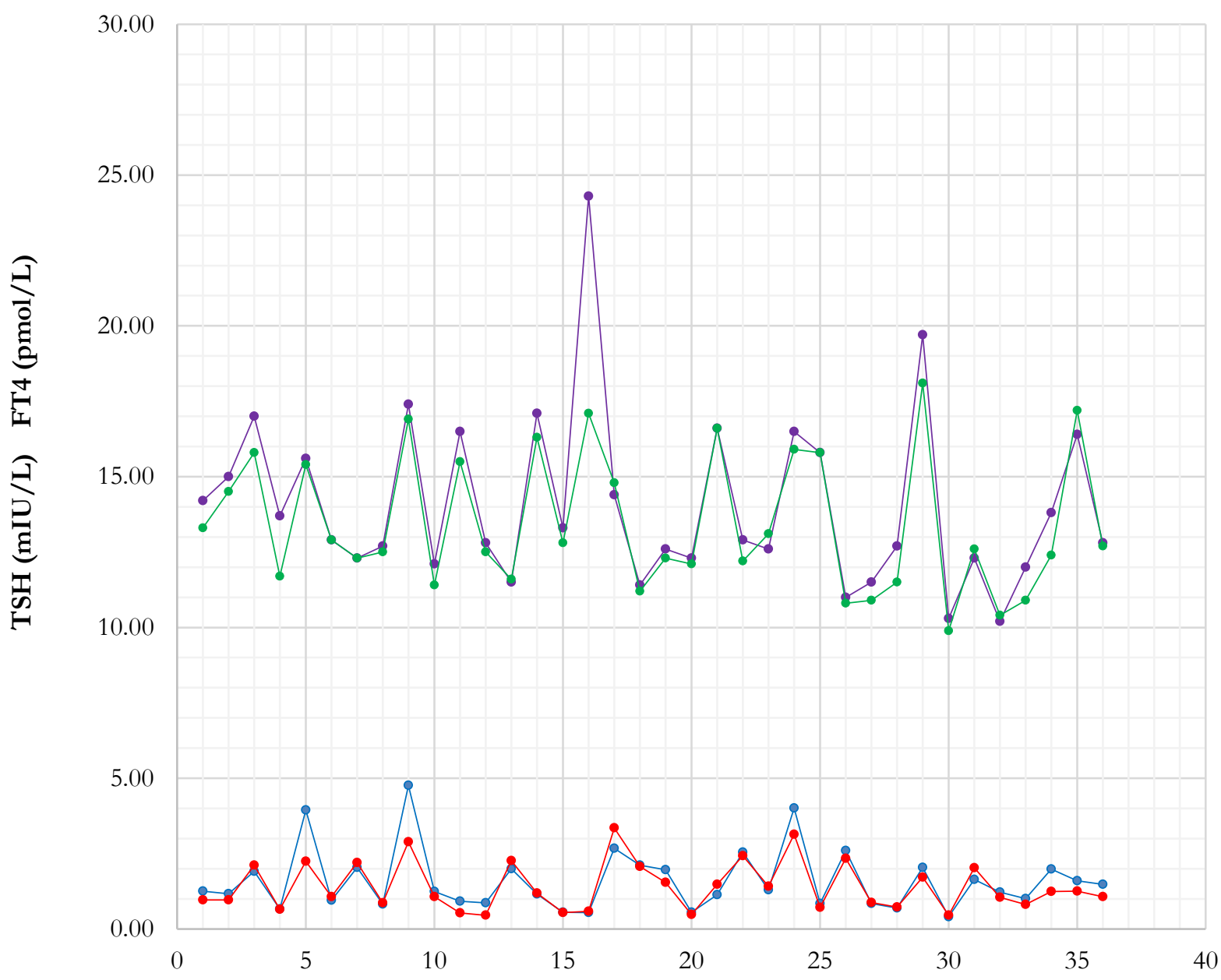

Reference Number
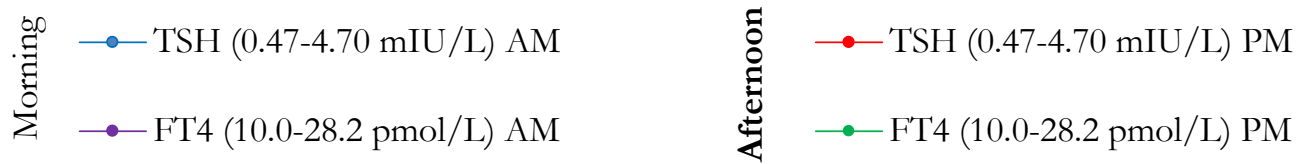
Figure 2-Boxplots of TSH and $\mathrm{FT}_{4}$ morning and afternoon values

\section{Before $\log _{10}$ Transformation}
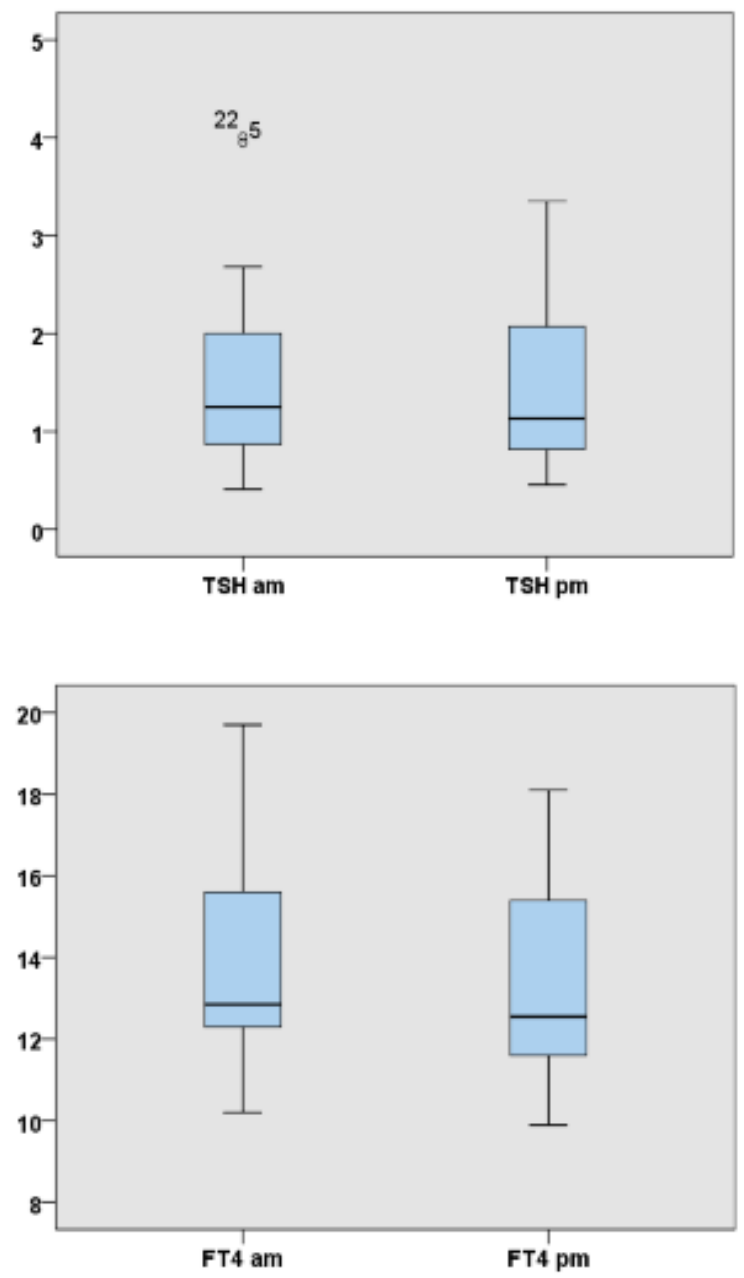

Results

A total of $36(n=36)$ individuals were enrolled for this study. They were categorized in to three age groups (age 21 to 30 , age 31 to 40 and age 41 to 50) and equal number of males and females were selected for each age group as it represented healthy adult population.

Figure 1 shows the diurnal changes of each observed values for both $\mathrm{TSH}$ and $\mathrm{fT}_{4}$. Figure 2 illustrates the distribution of $\mathrm{TSH}$ and $\mathrm{fT}_{4}$ values, morning and afternoon, after removing outliers. This reveals the changes of distribution before and after $\log _{10}$
After $\log _{10}$ Transformation
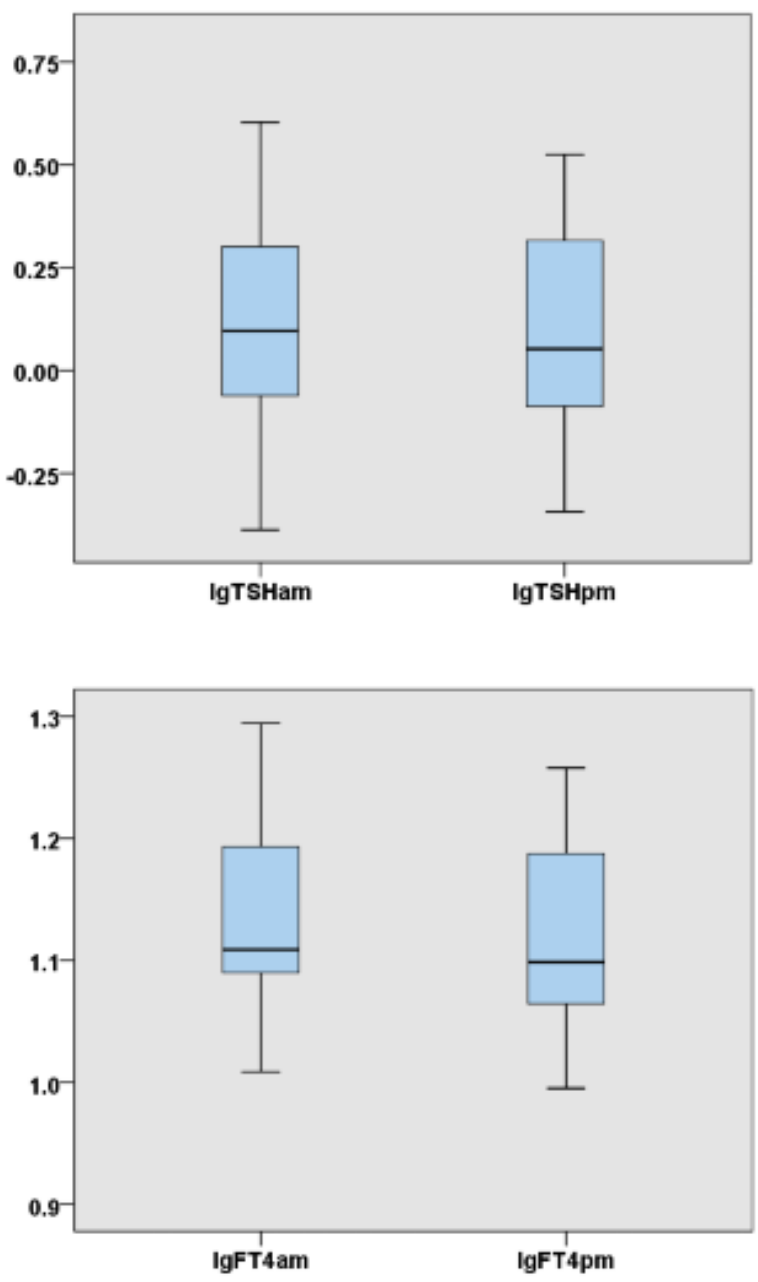

transformation. All the data obtained from morning and afternoon TSH and $\mathrm{fT}_{4}$ values were analysed to find whether the data were normally distributed. Basic statistical analysis revealed that the skewness and kurtosis values were $>+1.000$ for morning values of both TSH and $\mathrm{fT}_{4}$ and kurtosis value for afternoon $\mathrm{fT}_{4}$ was $<-1.000$. Normality tests were performed to assess the normal distribution of observed data, and that results also revealed the data sets were not normal.

Therefore the, Resistant Rules for Outlier Labelling was applied to identify possible outliers; two outliers were detected and removed from the data set (11). Remaining 
34 results were used for further analysis. Normality test was applied for the remaining data $(n=34)$, and still the data set deviated from normal distribution. But basic statistical analysis of the remaining data revealed that, only the skewness and kurtosis values of TSH morning data, remained $>+1.000$, whereas all other three parameters were within +1.000 and -1.000 .

Remaining 34 values were analysed to find whether there is any significant variation between TSH and fT4 values with gender and with age groups. The results of Independent Samples Test for Gender and ANOVA analysis for three age groups revealed that the variations of TSH and $\mathrm{fT}_{4}$ with gender or with age group were not significant. All the $p$-values were greater than $p=0.05$ at $95 \%$ confidence interval level. As there is no significant variation of $\mathrm{TSH}$ and $\mathrm{fT}_{4}$ values with gender or with age groups, the results were analysed for diurnal variation. Before applying paired $T$-test to find diurnal variation the morning and afternoon data, they were analysed to check whether there is any significance difference between the group variances by applying $F$-test (12).

Calculated F-values for both TSH (1.2800) and $\mathrm{FT}_{4}$ (1.0811) are lower than critical $F$-value (1.84), which were obtained from $F$-table at 95\% confidence interval level. Because of that the null hypothesis is accepted as true which is that, there is no significant difference between the variances. So paired $T$-test can be applied directly for remaining morning and afternoon TSH and $\mathrm{FT}_{4}$ values $(n=34)$. Table 2 shows the results of paired T-test for TSH and $\mathrm{FT}_{4}$, morning and afternoon data.

A statistically significant difference was observed only for $\mathrm{fT}_{4}$ morning and afternoon data. $P$-values obtained from paired $T$-test for fT4 $(p<0.001)$ are lower than $p=0.05$ at $95 \%$ confidence interval level, and $p$-value is higher than $p=0.05$ for TSH $(p=0.052)$. Even though the F-test results showed no significant difference between the variances, results of skewness and kurtosis of morning TSH and $\mathrm{fT}_{4}$ data, and both tests of normality for $\mathrm{TSH}$ and $\mathrm{fT}_{4}$ morning and afternoon data, were not normally distributed, as all the $p$-values for normality tests were lower than $p=0.05$ at $95 \%$ confidence interval level.

Therefore, the data for both TSH and fT4 $(n=34)$ were $\log$ transformed to assess whether the values were normally distributed. The $\log$ transformed values were analysed to assess the significance of diurnal variation of both TSH and $\mathrm{fT}_{4}$ values. After $\log _{10}$ transformation, the skewness and kurtosis values of morning TSH and $\mathrm{fT}_{4}$ levels were within +1.000 to -1.000 , which indicated the data sets were normally distributed.

The normality test results after $\log _{10}$ transformation, revealed that both tests of normality for TSH morning and afternoon data were normally distributed, as all the $p$ values were higher than $p=0.05$ at $95 \%$ confidence limits. Even after $\log _{10}$ transformation, only Shapiro-Wilk test gave results as normally distributed, for $\mathrm{fT}_{4}$ morning and afternoon data, whereas Kolmogorov-Smirnova test with Lilliefors Significance Correction indicate the data set still deviated from normal distribution.

After $\log _{10}$ transformation, remaining $(n=34)$ values were analysed to find whether there is any significant variation between TSH and $\mathrm{fT}_{4}$ values with gender and with age groups. The results of Independent Samples Test for Gender and ANOVA analysis for three age groups revealed that the variations of $\log \mathrm{TSH}$ and $\log \mathrm{fT}_{4}$ with gender or with age group were not significant. All the $p$ values are greater than $p=0.05$ at $95 \%$ confidence limits.

Table 2: Paired Samples $T$-test for TSH and FT4, morning and afternoon data

$\begin{array}{cccc}\text { Pair } & \mathbf{t} & \text { df } & \text { Sig. (2-tailed) } \\ \text { TSH } \text { am - TSH pm } & 2.013 & 33 & 0.052 \\ \text { FT4 } \text { am - FT4 pm } & 4.034 & 33 & 0.000\end{array}$


As there is no significant variation of $\mathrm{TSH}$ and $\mathrm{fT}_{4}$ values with gender or with age groups even after $\log _{10}$ transformation, the results were analysed for diurnal variation. Before applying paired $T$-test to find diurnal variation of the morning and afternoon $\log _{10}$ transformed data, they were analysed to check whether there is any significant difference between the group variances by applying $F$-test (12).

Calculated $F$-values for both $\log \mathrm{TSH}(1.0566)$ and $\operatorname{logf\mathrm {T}_{4}}$ (1.0051) were lower than critical $F$-value (1.84), at the 95\% confidence interval level. Thus, paired $T$-test was directly for $\log _{10}$ transformed morning and afternoon
TSH and $\mathrm{fT}_{4}$ values $(n=34)$. Table 3 shows that a statistically significant difference is observed for both TSH and $\mathrm{fT}_{4}$ morning and afternoon data, after $\log _{10}$ transformation. $P$-values obtained from paired $T$-test for $\log \mathrm{TSH}(p=0.013)$ and $\log \mathrm{FT}_{4}(p<0.001)$ are lower than $p=0.05$ at $95 \%$ confidence interval level.

Diurnal variation observed in this study is applicable only for a healthy population and is statistically significant. However, the observed values were found within the manufacturer's reference limits for both TSH \& fT4. (Table 4)

Table 3: Paired Samples $T$-test for $\log$ TSH and $\operatorname{logFT4}$, morning and afternoon data

Pair

LogTSHam - logTSHpm

$\operatorname{logFT4am~-~} \log$ FT4pm $\mathbf{t}$

2.616

4.118 df

33

33
Sig. (2-tailed)

0.013

0.000

Table 4: Comparison of reference ranges of the study with that of the manufacturer

TSH (mIU/L)
Reference Range*

0.41 to 4.01

0.45 to 3.35

0.47 to 4.70
1.54

1.39

Manufacture's Values
1.48
FT4 (pmol/L)

\begin{tabular}{|c|c|c|c|c|}
\hline Study (am) & 1.54 & 0.41 to 4.01 & 12.9 & 10.2 to 19.7 \\
\hline Study (pm) & 1.39 & 0.45 to 3.35 & 12.6 & 9.9 to 18.1 \\
\hline Iufacture's Values & 1.48 & 0.47 to 4.70 & 15.9 & 10.0 to 28.2 \\
\hline
\end{tabular}

\footnotetext{
* Upper and lower values of observed data of the study were given as reference ranges.
} 
$\log$ TSHam

\section{Pearson Correlation}

Sig. (2-tailed)

$\mathbf{N}$

Pearson Correlation

Sig. (2-tailed)
0.917

0.317

0.000

0.068

34

34

0.167

0.959

0.344

0.000

$\mathbf{N}$

34
There was a strong, significant and positive correlation between morning and afternoon $\log \mathrm{TSH}$ data, and also between morning and afternoon $\log \mathrm{T}_{4}$ data. However, when the $\log \mathrm{TSH}$ and $\operatorname{logf\mathrm {T}_{4}}$ were considered, there was a poor correlation between the values of the two hormones, which were not significant (Table 5).

\section{Discussion}

Studies vary in their conclusions regarding the diurnal variation of $\mathrm{TSH}_{\mathrm{H}}$ and $\mathrm{fT}_{4}$. The current evidence supports that the highest TSH level is observed in midnight and early morning, whereas the lowest is seen in the evening $(3,4)(10,11)$. There is a similar tendency for $\mathrm{fT}_{4}$ as well [7] though short-lived fluctuations of $\mathrm{fT}_{4}$ are observed throughout the day (9).

This is the first study of this nature done in Sri Lanka to observe the diurnal variation in TSH and fT4. No variation was observed in hormone levels due to age or sex, both before and after $\log _{10}$ transformation. Free thyroxine values showed a statistically significant diurnal variation $(\mathrm{p}<0.001)$, whereas diurnal variation of TSH showed no statistical significance $(\mathrm{p}=0.052)$ prior to $\log _{10}$ transformation.

TSH and fT4, showed a statistically significant difference for both TSH $(p=0.013)$ and fT4 $(p<0.001)$ values for the morning and afternoon after $\log _{10}$ transformation the study population was derived from two state institutions and therefore might have introduced a sampling bias.
The results observed is dependent on the performance characteristics of the analytical method used; immunochemiluminescence technology. The applicability of the study findings need to be verified by repeating the study in a general healthy population using other immunoassay techniques and platforms as well. In addition, the study should be extended to patients with thyroid dysfunction, and individuals of other age groups to investigate the pattern of diurnal variation in those sub groups.

However, as the study showed a statistically significant variation for both TSH and $\mathrm{fT}_{4}$ following $\log$ transformation, for morning and afternoon samples, it's best to collect samples at a defined time period in the day, preferably morning, to minimize the changes in test results due to diurnal variation. This needs to be considered during repeat and follow-up testing especially. Establishing reference ranges for TSH and $\mathrm{fT}_{4}$ for morning and afternoon separately would also be a useful step.

\section{Conclusion}

There is a diurnal variation in log transformed TSH and fT4 values of healthy adults from $20-50$ years and it is statistically significant at $95 \%$ confidence limits. Therefore, standardization of sample collection time may be important for $\mathrm{TSH}_{\mathrm{H}}$ and $\mathrm{fT}_{4}$ hormone assays. However, further studies are needed in patients with thyroid diseases and individuals of all age groups to ascertain the clinical significance of the diurnal variation observed. 
1. Kumar P, Clark M. Kumar and Clark's Clinical Medicine. $7^{\text {th }}$ ed. Elsevier Saunders; 2009. Endocrine Diseases; Chapter 18, pp 982

2. Marshall WJ, Bangert PK. Clinical Chemistry. 7th $^{\text {th }}$ : Elsevier Mosby; 2004. The Thyroid Gland; Chapter 9, pp 161-76.

3. Barman SM, Boitano S, Brooks HL, Barrett KE. Ganong's Review of Medical Physiology. 23 ${ }^{\text {rd }}$ ed: The McGraw-Hill; 2010. The Thyroid Gland; Chapter 20.

4. Burtis CA. Ashwood ER, Bruns DE. Tietz - Textbook of Clinical Chemistry \& Molecular Diagnosis. 4th ed: Elsevier Saunders; 2006. The Thyroid: Pathophysiology and Thyroid Function Testing; Chapter 52, pp. 2053-95.

5. VITROS Immunodiagnostic Products TSH Reagent Pack and TSH Calibrators. Instructions for Use. Ortho-Clinical Diagnostics - Johnson \& Johnson Company, 2010.

6. VITROS Immunodiagnostic Products FT4 Reagent Pack and FT4 Calibrators. Instructions for Use. Ortho-Clinical Diagnostics - Jobnson \& Jobnson Company, 2010.

7. Weeke J, Gundersen HJ. Circadian and 30 minutes variations in serum TSH and thyroid hormones in normal subjects. Acta Endocrinologica (Copenh). 1978 Dec: 80(4): pp 659-72.

8. Sviridonova MA, Fadeyev VV, Sych YP, Melnichenko GA. Clinical significance of TSH circadian variability in patients with hypothyroidism. Informa Healthcare. 2013: 38(1): pp 24-31.

9. Lucke C, Hehrmann R, von Mayersbach K, von zur Mühlen A. Studies on circadian variations of plasma TSH, thyroxine and triiodothyronine in man. Acta Endocrinologica (Copenh). 1977 Sep; 86(1), pp 81-8.

10. Weeke J, Laurberg P. Diurnal TSH Variations in Hypothyroidism. The Journal of Clinical Endocrinology and Metabolism. 1976 July; 43(1): pp 32-37.

11. Hoaglina DC, Iglewiczb B, Tukey JW. Performance of Some Resistant Rules for Outlier Labelling. Journal of the American Statistical Association. 1986; 81(396): pp 991-9.

12. Kaplan LA, Pesce AJ. Clinical Chemistry. $5^{\text {th }}$ ed. St. Louis, Missouri: Mosby Elsevier, 2010. Laboratory Statistics; Chapter 23, pp 415-38. 